\title{
Aspectos de la sintaxis del Rromané de Chile en textos orales tradicionales (1)
}

\author{
Álvaro Javier González Concha** \\ Gastón Felipe Salamanca Gutiérrez ${ }^{* * *}$
}

\section{Resumen}

Este trabajo, dividido en dos artículos, presenta los aspectos más sobresalientes de la sintaxis del rromané hablado en Chile, presentes en tres textos orales tradicionales (paramíchura). En este primer artículo, se presentan los aspectos principales de la morfología de esta lengua, la metodología utilizada en esta investigación, el marco de referencia al que se ciñe (la Tagmémica) y, como foco, los tipos de Frase Modificada Sustantiva (FMS) y Frase Modificada Verbal (FMV), con sus respectivos tagmemas Centro y Modificador. No se incluye aquí un apartado de conclusiones, porque éstas se contienen en el segundo artículo.

Palabras clave: Rromané, sintaxis del rromané, gitanos, tagmémica.

\section{Aspects of the chilean Rromané sintax on traditional oral texts}

\begin{abstract}
The following work, divided into two articles, introduces the most outstanding aspects of the syntax of the Romani spoken in Chile, which are found in three traditional oral texts (paramichura).

In this first article we introduce the main aspects of this language morphology, the methodology used for this research, the referential frame chosen for the data analisis (the tagmemics pattern), and, as the main focus, the types of Modified Noun Phrase (MNPh) and Modified Verb Phrase (MNPh) found in the texts studied with their corresponding Central and Modifying tagmemes. We do not include here the conclusions because they are contained in the second article.
\end{abstract}

Key words: Romani, Romani Syntax, gypsies, tagmemics.

\footnotetext{
Este artículo y el siguiente se insertan en el Proyecto "Sintaxis del rromané", Código: 034320 3/I de la Universidad del Bío-Bío. Para las palabras en rromané utilizamos el grafemario propuesto por Salamanca y Lizarralde (2008).

** Doctor en Lingüística. Universidad del Bío-Bío. algonzal@ubiobio.cl

*** Doctor en Lingüística. Universidad de Concepción. gaston.salamanca@gmail.com
} 


\section{Introducción}

En Chile existen descripciones etnográficas, fonológicas y morfológicas de la lengua de los gitanos rrom (Salamanca y González, 1999; González y Salamanca, 2001; Salamanca, 2003; 2004; 2005; 2006 y 2008; y Lizarralde y Salamanca, 2010), las cuales constituyen los únicos estudios lingüísticos sobre el romani de este país.

Desde el punto de vista de la vitalidad de su lengua y cultura, los gitanos de Chile se encuentran en una situación privilegiada, por cuanto conservan gran parte de sus costumbres; de hecho, Chile es uno de los pocos países en Sudamérica en el cual una cantidad importante de gitanos aún son nómades. Esta situación repercute positivamente en el mantenimiento de su lengua, cuestión que no deja de sorprender, ya que el número total de gitanos en nuestro país se calcula en no más de 8.000 (Sotomayor, 1993).

El objetivo general en esta investigación es analizar la sintaxis del rromané de Chile en tres textos orales tradicionales. Dada la extensión que esto implica -no obstante ser ésta una presentación panorámica de este nivel lingüístico- es que hemos dividido este trabajo en dos artículos.

Este primer artículo presenta los aspectos más prominentes de la morfología nominal y verbal de esta lengua; algunos aspectos focales del marco de referencia que hemos seguido (la Tagmémica); la metodología seguida paso a paso para arribar a la clasificación que presentamos; y, finalmente, los tipos de Frases Modificadas Sustantivas (FMS) y Frases Modificadas Verbales (FMV), con sus respectivos tagmemas Centro y Modificador. No se incluye aquí un apartado "Conclusiones", pues se presenta en el segundo artículo.

En el segundo artículo, no se especifica el marco de referencia seguido, ni la metodología utilizada, pues éstos coinciden con los que se presentan en esta primera parte. Así, se detallan inmediatamente los tagmemas que ocurren en la Frase Modificada Adjetiva (FMAdj), Frase Modificada Adverbial (FMAdv), Frases Seriadas (FSeriada), Frases Aposicionales (FApos) y Frase Conector-Eje (FC-Eje), así como los tagmemas que ocurren en el nivel de la Oración y los distintos tipos de éstas. Incluye, también, el apartado Conclusiones, en el cual se contienen los aspectos más prominentes de la descripción del Nivel de la Frase y de la Oración, una evaluación del marco de referencia escogido y posibles proyecciones del trabajo realizado. 
En este trabajo no se desarrolla una discusión teórica respecto del marco de referencia adoptado o de aspectos puntuales de la sintaxis del rromané, sino que se entrega una descripción de los aspectos más prominentes de la sintaxis de esta lengua, de modo que se pueda acceder de la manera más expedita posible a los datos. En esto seguimos a Salas:

La orientación de este análisis es descriptivista. Por supuesto, ninguna descripción es concebible en un vacuum teórico y metodológico, pero la intención fundamental de este trabajo no es discutir una teoría lingüística ni diseñar un cuerpo de procedimientos analíticos, ni establecer una doctrina gramatical universal[ ... ]lo que se quiere es describir adecuadamente los datos. En este sentido se puede decir que hay una posición epistemológica empiricista, y más estrictamente, empiricista funcionalista[...](Salas, 1978: 5)

En este contexto, aunque hay consciencia de lo enriquecedor que resulta discutir conceptos basales de la tradición gramatical, como son los de Cláusula, Oración o Proposición ${ }^{1}$, en esta tesis no se abundará en argumentos que apoyen o desaconsejen su utilización, sino que se adoptará uno de ellos -en este caso el de Oración- como una noción operativa que permite una descripción y análisis adecuados de los datos.

Finalmente, nos sumamos a lo expresado por Lizarralde y Salamanca (2010: 110), en cuanto a que "En el contexto de los escasos estudios relativos a la lengua hablada por los gitanos de Chile, nos parece que el presente trabajo aborda un objeto de estudio novedoso y constituye un aporte a la descripción lingüística". En efecto, este es el primer trabajo sobre la sintaxis del rromané jorajané hablado en Chile y, por lo mismo, constituye una contribución relevante a su estudio.

\section{El Romani}

La lengua de los gitanos (el romani) pertenece a la familia de las lenguas indoeuropeas, dentro de las que se encuentran el hindi, el gujarati y el mahrata, entre otras. Aunque es una misma lengua en todo el mundo, tiene variaciones dialectales que los mismos gitanos reconocen.

El romani (o rromané, como la denominan los gitanos en Chile) es una lengua inflexional, preferentemente sufijada, que ha mantenido una

1 Gutiérrez (1997), por ejemplo, contiene en el segundo y tercer capítulos una discusión interesante respecto de los conceptos de Cláusula, Proposición y Oración, y se pregunta si es necesario la utilización de este último. 
parte de su léxico tradicional, con una flexión también tradicional, y otra parte -equivalente a los préstamos léxicos- con una flexión distinta, propuesta de análisis planteada, entre otros, por Hancock (1993), para la morfología del romani de Estados Unidos, y confirmada por Salamanca (2003) para el rromané de Chile.

La huella del periplo seguido por esta lengua se puede encontrar en las palabras de origen iraní presentes en casi todas sus variantes dialectales. A modo de ejemplo: baxt ('suerte', 'fortuna'), ambrol ('pera'), khangeri ('iglesia'), angustri ('anillo'), vurdon ('carro'), zor ('fuerza'), cikat ('frente'). Algunas palabras de origen armenio son: bov ('horno') y grast ('caballo'). Las siguientes son de origen griego: drom ('camino'), karfin ('clavo'), klidi ('llave'), kokalo ('hueso'), papin ('ganso'), petalos ('herradura'), zumi ('sopa'), e isviri ('martillo') (Soravia, 1984: 22).

Actualmente, la mayoría de los grupos gitanos habla algún dialecto del romani. Una excepción a esta tendencia la constituye el grupo caló de España, el que sufrió un grado de desmantelamiento tal que sólo mantiene el léxico de la lengua. Otra excepción la constituye el grupo ludár, el cual perdió el romani y actualmente habla un dialecto rumano antiguo.

\subsection{Morfología ${ }^{2}$}

\subsubsection{Morfología de ítemes tradicionales (temáticos) y no tradicionales (atemáticos)}

Salamanca (2003: 26-27), hace referencia a la morfología temática y atemática del rromané hablado en Chile. En esto coincide con otros lingüistas que han estudiado otras variantes de esta lengua (Hancock, 1993; Grant, 1994). Esta distinción se establece por las diferencias que se producen en la declinación de los nombres y en la conjugación de los verbos originarios de India y de los préstamos posteriores que tienen su origen en las lenguas de Los Balcanes y en otras lenguas europeas. Al respecto, Grant señala:

The most important distinction to be made in Romani is that between thematic and athematic grammar. Stem formation, derivational and some inflectional morphology in certain dialects (eg. Past participle and affixes facilitating adoption of loan verbs) separate inhereted words or old loans (pre-

2 Por restricciones de espacio, no nos referimos aquí a aspectos de la fonología del rromané. Éstos se pueden ver en González y Salamanca (2001). 
European words and some early Greek loans) from new loans. Thus Vlax dialects make a distinction between thematic morphology and athematic morphology. Athematic morphology applies to loan words and derives largely from Greek. (Grant, 1994: 5)

Sobre la morfología del rromané hablado en Chile, Salamanca (2003, 2004 y 2008) entrega un análisis exhaustivo de la morfología del sustantivo y de la morfología del verbo.

\subsubsection{Morfología nominal}

a) Género: existen dos géneros: masculino y femenino; todos los sustantivos pertenecen a alguna de estas dos clases. Sin embargo, sólo algunos lo marcan morfológicamente. Por ejemplo:

$$
\begin{aligned}
& \text { basn-ó 'chancho' } \\
& \text { basn-í 'chancha' }
\end{aligned}
$$

En otros casos, los sustantivos no presentan esta variación morfológica; sin embargo, su concordancia con el género y número del artículo o del adjetivo permite determinar al género que pertenecen. Tal es el caso de la palabra "rat" ('sangre'), cuya concordancia con el artículo y adjetivo masculinos nos permite determinar que éste es su género:

o rat si loló 'la sangre es roja'.

b) Número: Hay dos números gramaticales: singular y plural, tal como se aprecia en el siguiente ejemplo:

$$
\begin{aligned}
& \text { yakh 'ojo' } \\
& \text { yakhá 'ojos' }
\end{aligned}
$$

El ejemplo anterior muestra, además, que el número singular, al igual que en español, no se marca en esta lengua.

c) Caso: se expresan morfológicamente los siguientes casos gramaticales:

Nominativo: Indica que el sustantivo funciona como Sujeto en la cláusula.

Acusativo: Indica que el sustantivo funciona como Objeto Directo en la cláusula.

Dativo: Indica que el sustantivo funciona como Objeto Indirecto en la cláusula. 
Ablativo: Indica que el sustantivo funciona en la cláusula como un Complemento Circunstancial de Origen o Causa.

Instrumental: Indica que el sustantivo funciona en la cláusula como un Complemento Circunstancial de Medio o Compañía.

Genitivo: Indica que el sustantivo funciona como un Complemento del Nombre.

Vocativo: Indica que el sustantivo cumple una función apelativa.

\subsubsection{Morfología verbal}

Existen verbos regulares (no modifican su raíz) e irregulares (modifican su raíz para los tiempos perfectivos e imperfectivos) (Salamanca, 2004: 75). En ambos casos las categorías gramaticales expresadas son tiempo/ aspecto y persona/número.

En rromané, se marcan las categorías de tiempo y aspecto de manera sincrética, y se distinguen tiempos imperfectivos y tiempos perfectivos. Los tiempos imperfectivos son el presente, el futuro y el pretérito imperfecto. Los tiempos perfectivos son el pretérito perfecto y el pretérito pluscuamperfecto.

Otra distinción importante es entre "tiempos básicos" y "tiempos derivados". Los tiempos "básicos" son el presente y el pretérito perfecto. Se les asigna este nombre, porque a partir de ellos se construyen el futuro, el pretérito imperfecto y el pretérito pluscuamperfecto, que son los tiempos derivados.

La persona y el número también se expresan de forma sincrética. Las personas gramaticales son: primera, segunda y tercera; los números, singular y plural.

También es destacable que el verbo "tener" se construye con el verbo "ser" más un clítico en caso acusativo.

\section{Marco de Referencia}

\subsection{Consideraciones preliminares}

Para esta investigación, se tomó como marco de referencia el enfoque tagmémico propuesto por Pike (1958) y aplicado y sistematizado posteriormente por Elson y Pickett (1964) en su libro An Introduction to morphology and Syntax. Este marco de referencia ha demostrado ser útil para el análisis y la descripción de los sistemas gramaticales de distintas lenguas. 
Dos aspectos que destacan en el enfoque tagmémico es que utiliza una misma metodología para los análisis morfológicos y sintácticos, y el hecho de que no inicia el análisis con una división dicotómica. En efecto, tradicionalmente la diferencia entre morfología y sintaxis ha creado una brecha que ha tenido como consecuencia que los estudios de estas materias se hayan realizado con metodologías separadas. Pike cuestiona esta división entre morfología y sintaxis, y considera a la palabra como un nivel más en la jerarquía gramatical -por encima del nivel del Tema y por debajo del nivel de la Frase-, de modo que, postula, se puede estudiar con la misma metodología con que se estudia la estructura del Tema (aspecto tradicionalmente morfológico) y el nivel de la Frase y la Oración (aspectos tradicionalmente sintácticos).

Este modelo constituye un aporte a los estudios gramaticales, ya que al estar basado en una concepción serial de la gramaticalidad, se tiene la posibilidad de aplicar la técnica del "recurrente parcial", tanto a las oraciones como a las palabras. Para ello se introduce la noción de secuencia o cadena de constituyentes ("construcción").

\subsection{Conceptos básicos en Tagmémica}

\subsubsection{El Tagmema}

El tagmema es definido por Elson y Pickett como: “...the correlation of a grammatical function or slot with a class of a mutually subtitutable items occurring in that slot. This slot-class correlation has a distribution within the grammatical hierarchy of a language" (Elson y Pickett, 1964: 57).

Para comprender adecuadamente la noción de tagmema, conviene reparar en dos aclaraciones hechas por los autores: a) el término "casillero" (slot) refiere a la función gramatical del tagmema ("Sujeto", "Objeto", etc.); y b) el término "clase" (class) refiere a la lista de morfemas y secuencias de morfemas mutuamente sustituibles que instancian un casillero. De esta manera, la correlación del casillero Sujeto con la clase de ítemes sustituibles por otros en este casillero constituye el tagmema Sujeto.

En lo que dice relación con el análisis sintáctico, las construcciones han sido estudiadas en su mayor parte en términos de Constituyentes Inmediatos (CI). La tagmémica recoge esta noción; sin embargo, considera que la división binaria de la oración en Sujeto y Predicado puede no resultar del todo conveniente en los comienzos del análisis de una lengua no descrita (Cfr. Elson y.Pickett, 1964: 61). 
Por otro lado, el término "casillero" no se refiere exclusivamente o principalmente a la posición lineal en la cual se encuentran los morfemas o secuencias de morfemas, pues a) puede haber tagmemas discontinuos, como el tagmema Predicado en inglés (is Mary going?) y b) una función gramatical simple puede tener más de una posición, como el casillero Tiempo en español ("Pedro trabajará mañana" y "mañana trabajará Pedro").

La expresión "...has a ditribution within grammatical hierarchy of a language" hace referencia al hecho de que los tagmemas pueden ser parte de construcciones en algún nivel de la jerarquía gramatical. De acuerdo con Elson y Pickett, el término "jerarquía gramatical" "... refers to the fact that sequence of morphemes (analizable in terms of strings or tagmemes) may themselves manifest a single tagmeme...the tagmemes analyzed at each significant level constitutes the hierarchy of a language." (1964: 58)

De esta forma, lo fundamental está en la posibilidad de que una cadena de tagmemas pueda manifestar un tagmema único, que a su vez es parte de una construcción en un nivel superior de la jerarquía. Los tagmemas analizados en cada nivel significativo constituyen la jerarquía de una lengua, por lo tanto se concibe una gramática estructurada en términos de complejidad estructural creciente: palabra - frase - oración - período oracional - párrafo - texto.

\subsubsection{Niveles}

El enfoque tagmémico considera los siguientes Niveles: Tema, Palabra, Frase, Cláusula y Oración, ordenados en una jerarquía de menor (Tema, Palabra, Frase) a mayor (Cláusula, Oración). Es recomendable reconocer estos niveles jerárquicos en los que tagmemas y construcciones pueden ser identificados y descritos para dar mayor claridad al análisis. No obstante lo anterior, no todos los niveles recién mencionados serán significativos en cada una de las lenguas. Así, en el mixteco, por ejemplo, no se hace necesaria la distinción entre Palabra y Frase.

En algunas lenguas se hace necesario analizar el "tema" como un nivel separado, pero no en otras. En nuestro análisis, se asumió una postura más bien cauta, y se esperó a ver el funcionamiento del rromané para decidir si todos o sólo algunos de estos niveles serían considerados.

Por otro lado, cualquier secuencia de unidades que manifieste un tagmema será analizada como una construcción en un nivel diferente de ese en el que el tagmema es reconocido. Por ejemplo: 'el hombre bueno' 
es reconocido en el nivel de la Cláusula (manifiesta el tagmema Sujeto); sin embargo, es analizado en el nivel de la Frase. Por otro lado, una secuencia de morfemas que instancia un casillero se dice que manifiesta un tagmema en el nivel donde el casillero es descubrible.

Como ya se mencionó, el análisis tagmémico mantiene la visión tradicional de niveles distintos en la jerarquía gramatical. Ahora, si bien se distingue un orden en los niveles (Palabras, Frases, Oraciones) no se debe pensar que las oraciones van a estar construidas exclusivamente de frases, las frases exclusivamente de palabras, y así sucesivamente. Siempre existirá la posibilidad de que en esta jerarquía haya saltos, reinstalaciones, o retrocaídas, como se muestra en el siguiente esquema que presentan Elson y Pickett (1964: 86).

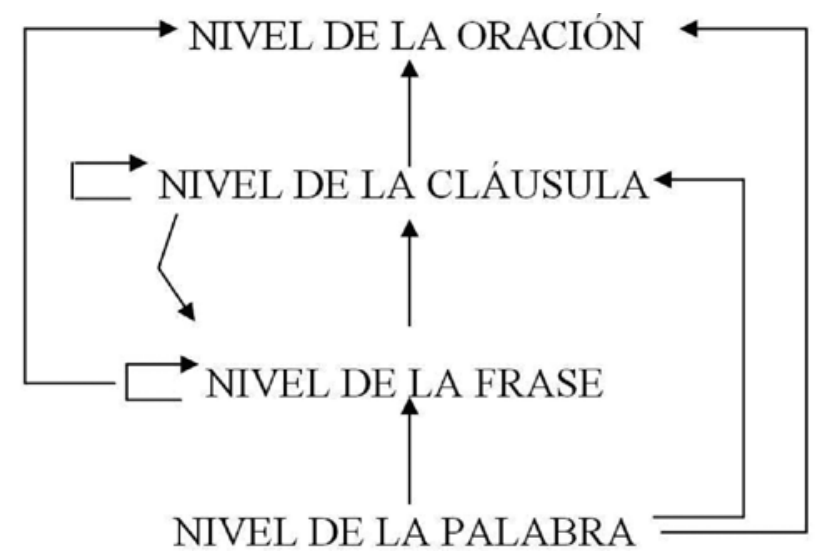

En este esquema, las flechas deben leerse como sigue: una secuencia de morfemas en el nivel $\underline{x}$ ("cola" de la flecha) instancia casilleros en el nivel $¥$ (cabeza de la flecha). Las flechas en el centro indican la distribución más común, que nos dice que lo más habitual es que las construcciones de un nivel determinado instancien casilleros del nivel inmediatamente superior. Por lo tanto, se espera que los constituyentes típicos de las cláusulas sean las frases; sin embargo, los fenómenos de traslapos y retrocaídas son muy comunes. En español, por ejemplo, cláusulas pueden instanciar casilleros en el nivel de la Frase, fenómeno llamado "retrocaída" en tagmémica.

\subsubsection{Construcciones}

Si nos detenemos en el hecho de que "A tagmeme may be manifested by a morpheme sequence which in turn is analyzable in terms of tagmemes." (Elson y Pickett, 1964: 58), podemos apreciar que cada tagmema puede 
ser analizado como una construcción compuesta, a su vez, de tagmemas. Como consecuencia de lo anterior, tenemos que no todos los morfemas en una secuencia dada se relacionan de la misma manera entre sí. Por ejemplo, en el enunciado la niña hermosa tomó té, la relación entre los morfemas que componen la frase la niña hermosa es mucho más fuerte y estrecha que la relación que existe entre cualquiera de ellos y el morfema "-ó" (pret. perfecto).

Consideremos ahora el enunciado ese hombre invitó a la niña hermosa. Esta secuencia de morfemas, que instancia un casillero gramatical (por lo tanto, manifiesta un tagmema), y que es ella misma analizable en términos de tagmemas constituyentes, se dice que manifiesta una Construcción. Dicho de otra manera, una Construcción es una cadena (potencial) de tagmemas cuya secuencia de morfemas manifestadores instancia un casillero gramatical. En el caso de nuestro ejemplo, en el primer enunciado (la niña hermosa tomó té), la secuencia la niña hermosa instancia el casillero Sujeto y, en el segundo enunciado (ese hombre invitó a la niña hermosa), el de Objeto.

Construcciones posibles son:

Temas: Por ejemplo: temas nominales y verbales

Palabras: Por ejemplo: sustantivos y verbos

Frases: $\quad$ Por ejemplo: frases nominales y frases eje-relacionador

Cláusulas: Por ejemplo: cláusulas transitivas e intransitivas

Oraciones: Por ejemplo: oraciones coordinadas y subordinadas

\subsubsection{Constituyentes Nucleares y Satélites (marginales)}

Esta noción hace referencia al hecho de que en una secuencia de tagmemas algunos aparecen como más prominentes que otros en la misma secuencia. Éstos más prominentes (Nucleares) suelen ser los obligatorios; y los menos prominentes (Satélites), los opcionales.

Por ejemplo, el tagmema Predicado en el nivel de la Oración, o el tagmema Centro en el nivel de la Frase, son constituyentes Nucleares. El tagmema Locativo en la Oración, o el de Modificador en la Frase, en tanto, son Satélites. 


\section{Metodología}

\subsection{Informantes}

La selección de los informantes se realizó considerando dos criterios básicos: que fueran hablantes nativos del rromané y mayores de veinte años ${ }^{3}$.

Con respecto al trabajo de campo, en primer lugar se grabaron los cuentos a velocidad normal. Luego se grabaron a velocidad lenta y ultralenta. Después, se procedió a transcribir fonéticamente todo el material. Posteriormente, se realizaron sesiones de chequeo para despejar dudas en la transcripción. Una vez chequeda la transcripción fonética del material, se precedió a su transcripción fonémica y su escritura grafémica.

Después de realizadas estas transcripciones, se realizó un primer análisis de las estructuras sintácticas. Luego se llevaron a cabo entrevistas, en las cuales se le preguntó al informante por ciertas estructuras encontradas, y algunas modificadas, para observar si su reacción era de aprobación, extrañeza o rechazo. De esta manera se pudo comprobar qué estructuras resultaban propias y comunes en rromané, y cuáles eran inapropiadas o inadmisibles.

\subsection{Datos obtenidos}

Los datos considerados para esta investigación se obtuvieron de textos orales tradicionales y, además, de frases y oraciones de cotejo. Estas últimas estaban destinadas a corroborar la información obtenida de los textos tradicionales y a despejar dudas acerca de la constitución y funcionamiento de algunas estructuras sintácticas encontradas.

\subsection{Descripción de los pasos que se siguieron en el análisis}

\section{Primer paso}

En la definición de "tagmema", se incluye la noción de casillero funcional y la clase de formas que llena o instancia ese casillero. En este contexto, lo primero que se realizó fue inspeccionar las formas presentes en la lengua, y, con ayuda de la "traducción de informantes" y de la "traducción

3 Otros aspectos del trabajo de campo se realizaron de acuerdo con las recomendaciones contenidas en Samarín (1967). 
analítica", plantear conjeturas acerca de la función de cada palabra o grupos de palabras. Luego se confeccionó una carta que incluyó nombres tentativos para las funciones. Así, por ejemplo:

$\begin{array}{lllll}\text { a) o } & \text { rrom } & \text { cherél } & \text { páile } & \\ \text { El } & \text { gitano } & \text { hace } & \text { pailas } & \\ \text { b) me } & \text { dikhlém } & \text { but } & \text { lulunllá } & \\ \text { yo } & \text { vi } & \text { muchas } & \text { flores } & \\ \text { c) o } & \text { rrom } & \text { cherdá } & \text { yek } & \text { jiu } \\ \text { el } & \text { gitano } & \text { hizo } & \text { un } & \text { hoyo }\end{array}$

\section{Segundo paso}

El segundo paso consistió en etiquetar los constituyentes de las oraciones en términos de la función que cada uno parecía tener. Algunas letras en mayúscula sirvieron de símbolo para cada función: P (Predicado), S (Sujeto), $\mathrm{O}$ (Objeto $)^{4}$. Estas letras se escribieron sobre cada palabra o grupo de palabras que indicaba esas funciones. Lo importante fue establecer una carta de datos sobre la base de los casilleros identificados:

$\begin{array}{ccc}\mathrm{S} & \mathrm{P} & \mathrm{O} \\ \text { o rrom } & \text { cherél } & \text { páile } \\ \text { el gitano } & \text { hace } & \text { pailas } \\ \mathrm{S} & \mathrm{P} & \mathrm{O} \\ \text { me } & \text { dikhlém } & \text { but lulunllá } \\ \text { yo } & \mathrm{vi} & \text { muchas flores } \\ \mathrm{S} & \mathrm{P} & \mathrm{O} \\ \text { o rrom } & \text { cherdá } & \text { yek jíu } \\ \text { el gitano } & \text { hizo } & \text { un hoyo }\end{array}$

\section{Tercer paso}

El tercer paso consistió en hacer una carta y transferir los enunciados del rromané:

$\begin{array}{ccc}\text { S } & \text { P } & \text { O } \\ \text { o rrom } & \text { cherél } & \text { páile } \\ \text { me } & \text { dikhlém } & \text { but lulunllá } \\ \text { o rrom } & \text { cherdá } & \text { yek jíu }\end{array}$

Para cada casillero en la carta, se muestra una lista de instanciadores de esos casilleros.

A partir de la carta, se establecieron las clases identificadas. Según la tagmémica, la lista de instanciadores de cada casillero diferente puede

4 La correspondencia de todas las abreviaturas utilizadas aparece en 5.2. 
ser considerada una clase instanciadora; si dos casilleros distintos tienen los mismos instanciadores, serán considerados una sola clase con dos posiciones estructurales alternativas.

Como hemos dicho, las relaciones que un tagmema puede tener con una Construcción, según los distintos niveles identificados (construcciones en el nivel de la Frase o de la Oración), son dos: el tagmema es obligatorio (Nuclear) a la construcción, o es opcional a ella (Satélite). Esta relación es indicada en fórmulas por los símbolos $+\mathrm{y} \pm$, respectivamente.

\section{Cuarto paso}

Se nombraron las clases con la terminología de la gramática tradicional (sustantivo, modificador, etc.), para favorecer la lectura del análisis.

\section{Quinto paso}

Con los datos obtenidos, se propuso una fórmula que mostrara la serie o cadena de tagmemas descubiertos. Las fórmulas propuestas en el modelo se señalan de la siguiente manera: los dos componentes de un tagmema -casillero y clase- son simbolizados como términos que preceden y siguen a los dos puntos (:) en el siguiente orden: casillero: clase. La relación en que estos tagmemas ocurren respecto de la Construcción es simbolizada por $+\mathrm{y} \pm$.

Así, por jemplo, la fórmula para la oración o rrom cherdá yek jíu 'el gitano hizo un hoyo' es la siguiente:

\section{Oración $=+\mathrm{S}: \mathrm{FMS}+\mathrm{P}: \mathbf{v} \quad+\mathrm{O}:$ FMS \\ FMS $=+$ Lim: Art + Centro $:$ Sust}

Esto es, la Oración contiene un tagmema Sujeto obligatorio instanciado por una Frase Modificada Sustantiva; un tagmema Predicado obligatorio, instanciado por un verbo, y un casillero Objeto obligatorio, instanciado por una Frase Modificada Sustantiva. El signo "=" indica que la secuencia de tagmemas que constituyen la construcción que está a la izquierda del signo es explicada por los símbolos que están a la derecha de éste. En el nivel inmediatamente inferior, la Frase Modificada Sustantiva contiene un casillero Límite obligatorio, instanciado por un artículo ("o" y "yek", en este caso), más un tagmema Centro instanciado por un sustantivo obligatorio ("rrom" y "jiu", en este caso).

Para nuestra descripción, se utilizan básicamente esquemas, debido a que muestran con mayor claridad la noción de niveles. La estructura 
general, subyacente a cada esquema particular, es la que se presenta a continuación:

\begin{tabular}{|c|c|c|c|}
\hline Construcción: & \multicolumn{3}{|c|}{ O } \\
\hline Función: & S & $+\mathrm{P}$ & Obj dir \\
\hline Clase: & FMS & V & FMS \\
\hline & Tag.1 & Tag.2 & Tag.3 \\
\hline
\end{tabular}

Los esquemas para la oración mencionada anteriormente quedan de la siguiente manera:

\begin{tabular}{|l|l|l|}
\hline \multicolumn{3}{|c|}{$\mathrm{O}$} \\
\hline$+\mathrm{S}$ & $+\mathrm{P}$ & + Obj dir \\
\hline FMS & $\mathrm{V}$ & FMS \\
\hline
\end{tabular}

\begin{tabular}{|c|c|}
\hline \multicolumn{2}{|c|}{ FMS } \\
\hline +Lim & +Centro \\
\hline Art & Sust \\
\hline
\end{tabular}

\section{Sintaxis del Rromané de Chile}

\subsection{Consideraciones previas}

Presentamos a continuación la sistematización del análisis sintáctico de tres cuentos tradicionales gitanos (paramichura). La modalidad elegida fue la presentación a través de esquemas, para visualizar de mejor manera las relaciones jerárquicas. Omitimos aquí aquellos marcadores que pertenecen, más bien, al nivel de organización del relato y no a un nivel estrictamente sintáctico. Es el caso de ciertos usos de "bueno", "entonces", entre otros.

La traducción de las oraciones es semilibre. Hemos intentado mantener la estructura del rromané hasta donde ha sido posible, de modo que se encontrarán en el texto algunas glosas como "vio lo", "dijo le", etc. Sólo en aquellos casos donde la traducción literal atentaba de manera radical contra la gramaticalidad en español, ésta se ajustó, con el fin de facilitar su comprensión. Es el caso, por ejemplo, de merén bokhátar, cuya traducción literal es 'mueren hambre de'. En este caso preferimos ajustar la glosa y traducir como 'mueren de hambre'.

\subsection{Notación}

Las abreviaturas utilizadas en el análisis son las que siguen: 


\begin{tabular}{|c|c|}
\hline acus $=$ Acusativo & neg = Negación \\
\hline Adj = Adjetivo & num $=$ Numeral \\
\hline Adj sust $=$ Adjetivo sustantivado & O = Oración \\
\hline Adv $=$ Adverbio & Obj dir = Objeto directo \\
\hline Adv tpo $=$ Adverbio de tiempo & Obj ind $=$ Objeto indirecto \\
\hline Art def = definido & ODep = Oración Dependiente \\
\hline Art indef $=$ indefinido & OIndep = Oración Independiente \\
\hline Calif $=$ Calificativo & O interr = Oración interrogativa \\
\hline Conec $=$ Conector & O imp = Oración imperativa \\
\hline Conj coord = Conjunción coordinante & O excl = Oración exclamativa \\
\hline Conj cop = Conjunción copulativa & $+\mathrm{P}=$ Predicado obligatorio \\
\hline Conj subord = Conjunción subordinante & Part interr $=$ Partícula interrogativa \\
\hline Coord $=$ Coordinación & Part fut = Partícula de futuridad \\
\hline Cóp = Cópula verbal & Part Sub = Partícula de subordinación \\
\hline Cuantif $=$ Cuantificador & Pos $=$ Posesivo \\
\hline Descrip = descriptivo & Prep = Preposición \\
\hline Distrib. $=$ distributivo & Pron (ablat) = Pronombre ablativo \\
\hline $\mathrm{eP}=$ Predicado ecuacional & Pron $($ dat $)=$ Pronombre dativo \\
\hline eS = Sujeto ecuacional & Pron $($ dem $)=$ Pronombre demostrativo \\
\hline esp = especificativo & reit $=$ reiterativo \\
\hline excl = exclamativo & rel $=$ relativo \\
\hline FApos $=$ Frase aposicional & $S=$ Sujeto \\
\hline FC-Eje $=$ Frase conector-eje & $\mathrm{sP}=$ Predicado estativo \\
\hline FMAdj $=$ Frase modificada adjetiva & $\mathrm{sS}=$ Sujeto estativo \\
\hline FMAdv $=$ Frase modificada adverbial & Sust $=$ Sustantivo en caso nominativo \\
\hline FMS = Frase modificada sutantiva & Sust (genit) = Sustantivo en caso genitivo \\
\hline FMV = Frase modificada verbal & Sust (inst) $=$ Sustantivo en caso instrument \\
\hline FPrep = Frase preposicional & Sust $($ voc $)=$ Sustsantivo vocativo \\
\hline FScom $=$ Frase sustantiva complementaria & $\mathrm{V}=\mathrm{Verbo}$ \\
\hline FSeriada $=$ Frase seriada & Vmodal $=$ Verbo modal \\
\hline FSprincip = Frase sustantiva principal & Vprincipal = Verbo principal \\
\hline Intens $=$ intensificador & Vpronom $=$ Verbo pronominal \\
\hline Lím = Límite & Yuxtap = Yuxtaposición \\
\hline Loc $=$ Locativo & ( ) = elisión \\
\hline Mod $=$ Modificador & \\
\hline
\end{tabular}

\subsection{Nivel de la Frase}

En esta sección, sistematizamos los tipos de Frases encontrados y los Modificadores, con su posición respecto de la palabra que instancia el casillero Centro. Las Construcciones en el nivel de la Frase incluyen un tagmema Centro y, generalmente, pero no siempre, uno o más tagmemas Modificadores.

En el análisis del nivel de la Frase, sólo entregaremos una descripción de la estructura interna; no detallaremos los tagmemas que los distintos 
tipos de Frases instancian en el nivel de la Oración, pues éstos serán descritos en el segundo artículo.

\subsubsection{Frase Modificada Sustantiva (FMS) y sus Modificadores}

En rromané, un tipo de Frase Modificada es aquella cuyo casillero Centro es instanciado por un sustantivo o por un pronombre y que potencialmente puede incluir algún tagmema Modificador.

La construcción más típica que encontramos en este tipo de Frase es aquella que tiene un casillero Límite y un tagmema Centro instanciado por un sustantivo. Por ejemplo:

\section{o rrom}

'el gitano'

Su esquema es:

\begin{tabular}{|c|c|}
\hline \multicolumn{2}{|c|}{ FMS } \\
\hline Lím & Centro \\
\hline Art def & Sust \\
\hline o & rrom \\
\hline
\end{tabular}

Esta es una FMS que consiste en un casillero Límite obligatorio instanciado por un artículo (definido), más un casillero Centro obligatorio instanciado por un sustantivo. En la FMS cuyo Centro es un sustantivo, el casillero Límite es obligatorio, tanto para un sustantivo común como para un sustantivo propio ${ }^{5}$. Así, por ejemplo: o rakló dikhlá... 'el muchacho vio...', pero también: o Chirilo dikhlá... 'el Chirilo vio...' y $\boldsymbol{e}$ Lola dikhlá.... 'la Lola vio...'

Como instanciador del casillero Centro, puede aparecer también un pronombre personal en distintos casos gramaticales (nominativo, acusativo, dativo, etc.), como en el caso de la FMS de la siguiente oración:

\section{tu peradán e phabái}

'tú botaste la manzana'

El esquema es:

5 Cada vez que se le solicitó a los informantes que elicitaran una frase de este tipo -con o sin Límite en español- ésta tenía un casillero Límite. Al consultarles a los informantes acerca de la posibilidad de que no estuviera presente "o" en una frase como "o Chirilo", no manifestaron un rechazo terminante; sin embargo, nunca elicitaron de manera natural una frase de este tipo sin el casillero Límite. Por esta razón, se le considera obligatorio. 


\begin{tabular}{|c|c|c|c|}
\hline \multicolumn{4}{|c|}{$\mathrm{O}$} \\
\hline$S$ & $+\mathrm{P}$ & & \\
\hline FMS & \multirow{3}{*}{ V } & \multicolumn{2}{|c|}{ FMS } \\
\hline Centro & & Lím & Centro \\
\hline Pron & & Art def & Sust \\
\hline $\mathrm{tu}$ & peradán & $\mathrm{e}$ & phabái \\
\hline
\end{tabular}

Es interesante advertir que en rromané el artículo definido puede acompañar a un demostrativo, como en el caso de o gua 'el ese', situación que en español no es admisible, al menos en la norma estándar'.

Por ejemplo:

o gua marakhlá yek puñáva

'el ese encontró una alfombra'

\begin{tabular}{|c|c|}
\hline \multicolumn{2}{|c|}{ FMS } \\
\hline Lím & Centro \\
\hline Art def & Pron(dem) \\
\hline O & gua \\
\hline
\end{tabular}

De acuerdo con los textos analizados, una FMS puede instanciar tagmemas como Sujeto, Objeto Directo, Objeto Indirecto, Tiempo, Compañía, etc.

Los Modificadores que el tagmema Centro de una FMS puede tener en rromané son: Descriptivo, Cuantificador, Posesivo, Demostrativo, Límite y Oración Dependiente.

\subsubsection{Descriptivo}

En los cuentos analizados encontramos Modificadores Descriptivos tales como barí 'grande' y kalí 'negra'. Por ejemplo:

\section{yek pechín barí}

'una roca grande'

Su esquema es:

\begin{tabular}{|c|c|c|}
\hline \multicolumn{3}{|c|}{ FMS } \\
\hline Lím & Centro & Mod(Descrip) \\
\hline Art indef & Sust & Adj calif \\
\hline yek & pechín & barí \\
\hline
\end{tabular}

6 Expresiones como "el este", "la esta", etc., se pueden escuchar en ciertas variantes subestándar del español de Chile. 
Cuando ocurren simultáneamente un Modificador Demostrativo y uno Descriptivo, el Descriptivo se intercala entre el Demostrativo y el Centro, como en el ejemplo que sigue:

\section{gayá barí riat}

'esta gran noche'

Su esquema es:

\begin{tabular}{|c|c|c|}
\hline \multicolumn{3}{|c|}{ FMS } \\
\hline Mod(Dem) & Mod(Descrip) & Centro \\
\hline Adj dem & Adj calif & Sust \\
\hline gayá & barí & riat \\
\hline
\end{tabular}

Este tipo de tagmema Mod(Descriptivo) puede ser instanciado también, por una Frase Modificada Adjetiva (FMAdj). Por ejemplo:

yek rakhli but sukár

'una niña muy hermosa'

Su esquema es:

\begin{tabular}{|c|c|c|c|}
\hline \multicolumn{2}{|c|}{ FMS } \\
\hline Lím & Centro & \multicolumn{2}{|c|}{ Mod(Descrip) } \\
\hline \multirow{2}{*}{ Art indef } & \multirow{2}{*}{ Sust } & \multicolumn{2}{|c|}{ FMAdj } \\
\cline { 3 - 4 } & & Mod & Centro \\
\cline { 3 - 4 } & & Intensif & Adj calif \\
\hline yek & rakhlí & but & sukár \\
\hline
\end{tabular}

En el caso de la FMAdj (como tagmema Modificador de la FMS), la posición que tiene respecto del casillero Centro es la misma que tiene el adjetivo calificativo, presentando una movilidad también concomitante con matices semánticos.

\subsubsection{Cuantificador}

Algunos de los cuantificadores encontrados en los datos fueron: sa 'toda', desudúi 'doce' y trin 'tres'. Por ejemplo:

\section{desudúi chavrré}

'doce hijos'

Su esquema es:

\begin{tabular}{|c|c|}
\hline \multicolumn{2}{|c|}{ FMS } \\
\hline Mod(Cuant) & Centro \\
\hline Adj num & Sust \\
\hline desudúi & chavrré \\
\hline
\end{tabular}


La posición del Cuantificador, al igual que en español, es antecediendo al casillero Centro. En el caso del Cuantificador "sa" ('toda'), su aparición exige la presencia de un casillero Límite, el cual precede al casillero Centro, de modo que el Cuantificador es desplazado en una posición. Así se observa en una frase como sa e gallé ('toda la gente'), cuyo esquema es:

\begin{tabular}{|c|c|c|}
\hline \multicolumn{3}{|c|}{ FMS } \\
\hline Mod(Cuant) & Lím & Centro \\
\hline Adj indef & Art def & Sust \\
\hline sa & e & gallé \\
\hline
\end{tabular}

Una frase en la que el Cuantificador va pospuesto al casillero Centro -en este caso e gallé sa ('la gente toda')- es una secuencia aceptada; sin embargo, no es de uso común.

\subsubsection{Posesivos}

En el caso de los posesivos, aparecen los siguientes: chi 'tu', po 'su', mo 'mi'. Una frase en la que aparece un posesivo es la siguiente:

\section{chi korr \\ 'tu cuello'}

Su esquema es:

\begin{tabular}{|c|c|}
\hline \multicolumn{2}{|c|}{ FMS } \\
\hline Mod(Pos) & Centro \\
\hline Adj pos & Sust \\
\hline chi & korr \\
\hline
\end{tabular}

\subsubsection{Demostrativos}

En el caso de los demostrativos, encontramos ítemes como gayá 'esta'y gua 'ese'. Por ejemplo:

\section{gua chavrró}

'ese muchacho'

Su esquema es:

\begin{tabular}{|c|c|}
\hline \multicolumn{2}{|c|}{ FMS } \\
\hline Mod(Dem) & Centro \\
\hline Adj dem & Sust \\
\hline gua & chavrró \\
\hline
\end{tabular}

Los Demostrativos en rromané tienen siempre la misma posición (antecediendo al sustantivo), aunque tal como se ve en el ejemplo gayá 
barí riat 'esta gran noche', puede haber otro Modificador entre el demostrativo y el tagmema Centro.

\subsubsection{Límite}

Las clases de miembros que los manifiestan incluyen ítemes como: o 'el', yek 'un', e 'la'. Por ejemplo:

\section{e phabái}

'la manzana'

Su esquema es:

\begin{tabular}{|c|c|}
\hline \multicolumn{2}{|c|}{ FMS } \\
\hline Lím & Centro \\
\hline Art def & Sust \\
\hline $\mathrm{e}$ & phabái \\
\hline
\end{tabular}

\subsubsection{Oración Dependiente ${ }^{7}$}

En el nivel de la Frase, también podemos encontrar una Oración Dependiente como Modificador del casillero Centro. Por ejemplo:

\section{marakhlá yek puñáva kai fúrial}

'encontró una alfombra que vuela'

\begin{tabular}{|c|c|c|c|c|}
\hline \multicolumn{5}{|c|}{$\mathrm{O}$} \\
\hline$+\mathrm{P}$ & \multicolumn{4}{|c|}{ Obj dir } \\
\hline \multirow{4}{*}{ V } & \multicolumn{4}{|c|}{ FMS } \\
\hline & Lím & Centro & Mod & \\
\hline & \multirow[b]{2}{*}{ Art indef } & \multirow[b]{2}{*}{ Sust } & \multicolumn{2}{|l|}{ ODep } \\
\hline & & & $\frac{\text { S }}{\text { Suhordinador(Pron relat) }}$ & $+\mathrm{P}$ \\
\hline marakhlá & yek & puñáva & kai & fúrial \\
\hline
\end{tabular}

\subsubsection{Frase Modificada Verbal (FMV)}

En esta descripción consideramos como casos de FMV los siguientes:

5.3.2.1 Un verbo modal, más un verbo principal.

Un ejemplo de esta construcción es el siguiente:

mangén peravén e phabái e gallé

'quieren botar la manzana, las personas'

7 Más detalles sobre esta construcción se contienen en el segundo artículo. 
En este caso, el esquema es el siguiente:

\begin{tabular}{|c|c|c|c|c|c|}
\hline \multicolumn{2}{|c|}{ O } \\
\hline \multicolumn{2}{|c|}{$+\mathrm{P}$} & \multicolumn{2}{c|}{ Obj dir } & \multicolumn{2}{c|}{$\mathrm{S}$} \\
\hline \multicolumn{2}{|c|}{ FMV } & \multicolumn{2}{c|}{ FMS } & \multicolumn{2}{c|}{ FMS } \\
\hline Mod & Centro & Lím & Centro & Lím & Centro \\
\hline V modal & Vprincipal & Art def & Sust & Art def & Sust \\
\hline mangén & peravén & $\mathrm{e}$ & phabái & $\mathrm{e}$ & gallé \\
\hline
\end{tabular}

Esto es, una FMV que consiste en un Modificador, instanciado por un verbo modal, más un casillero Centro, obligatorio, instanciado por el verbo principal.

5.3.2.2 La secuencia: tagmema Modificador, partícula 'te', más tagmema Centro.

Un ejemplo de esta construcción es el siguiente:

\section{trubúl te kompañí amén}

'tienes que acompañar nos'

Su esquema es:

\begin{tabular}{|c|c|c|c|}
\hline \multicolumn{3}{|c|}{ O } & Obj dir \\
\hline \multicolumn{3}{|c|}{$+\mathrm{P}$} & \multirow{2}{*}{ Pron (acus) } \\
\cline { 1 - 2 } FMV & Centro & \\
\hline V modal & Partícula & Vprincipal & amén \\
\hline trubúl & te & kompañí & ampan
\end{tabular}

\subsubsection{La construcción del verbo "tener".}

Finalmente, consideramos como FMV la construcción del verbo "tener", esto es, el verbo "ser", más un pronombre en caso acusativo. Aquí se presentan dos casos:

a) Sólo con un pronombre acusativo (pospuesto). Por ejemplo:

e rromá si len kamionéte

'los gitanos tienen camionetas'

Su esquema es:

\begin{tabular}{|c|c|c|c|c|}
\hline \multicolumn{5}{|c|}{$\mathrm{O}$} \\
\hline \multicolumn{2}{|c|}{ S } & \multicolumn{2}{c|}{$+\mathbf{P}$} & Obj dir \\
\hline \multicolumn{2}{|c|}{ FMS } & \multicolumn{2}{c|}{ FMV } & FMS \\
\hline Lím & Centro & Centro & Mod & Centro \\
\hline Art def & Sust & V & Pron (acus) & Sust \\
\hline $\mathrm{e}$ & rromá & si & len & kamionéte \\
\hline
\end{tabular}


b) Con un pronombre acusativo pre y pospuesto. Por ejemplo:

\section{man si man but chavrré}

'tengo muchos hijos'

\begin{tabular}{|c|c|c|c|c|}
\hline \multicolumn{4}{|c|}{ O } & \multicolumn{2}{c|}{ Obj dir } \\
\hline \multicolumn{3}{|c|}{$+\mathrm{P}$} & \multicolumn{2}{c|}{ FMS } \\
\hline FMV & Centro & Mod & Mod(Cuant) & Centro \\
\hline Mod & V & Pron acus & Adj indef & Sust \\
\hline man & si & man & but & chavrré \\
\hline
\end{tabular}

En este caso, hay dos tagmemas modificadores instanciados por el pronombre acusativo man.

Una diferencia notoria con el español consiste en que en rromané el verbo "ser" es automáticamente omitido cuando se niega el verbo "tener" (del cual el verbo "ser" forma parte). Este comportamiento es sistemático y consistente. En efecto, si se niega una oración como la anterior (e rromá si len kamionéte 'los gitanos tienen camionetas'), la oración resultante es: e rromá (i)nai len kamionéte, cuya traducción literal es *'los gitanos no camionetas'. La elisión del verbo en una oración como ésta no es opcional, ya que si decimos * e rromá (i)nai si len kamionéte los informantes manifiestan rechazo y no la consideran una oración aceptable.

El esquema para la oración que nos ocupa es el siguiente:

\begin{tabular}{|c|c|c|c|c|c|}
\hline \multicolumn{6}{|c|}{$\mathrm{O}$} \\
\hline \multicolumn{2}{|c|}{$S$} & Negación & \multicolumn{2}{|c|}{$+\mathrm{P}$} & Obj dir \\
\hline \multicolumn{2}{|c|}{ FMS } & \multirow{3}{*}{ Adv neg } & \multicolumn{2}{|c|}{ FMV } & FMS \\
\hline Lím & Centro & & Centro & Mod & Centro \\
\hline Art def & Sust & & $(\mathrm{V})$ & Pron acus & Sust \\
\hline e & rromá & (i)nai & (si) & len & kamionéte \\
\hline
\end{tabular}

Otros ejemplos que encontramos en los relatos son los siguientes:

- (i)naj len charícha

neg. Obj dir3pl. carpita

no tienen carpita

Su esquema es:

\begin{tabular}{|c|c|c|c|}
\hline \multicolumn{4}{|c|}{$\mathrm{O}$} \\
\hline Negación & \multicolumn{2}{|c|}{$+\mathrm{P}$} & Obj dir \\
\hline \multirow{3}{*}{ Adv neg } & \multicolumn{2}{|c|}{ FMV } & FMS \\
\cline { 2 - 4 } & Centro & Mod & Centro \\
\cline { 2 - 4 } & (V) & Pron (acus) & Sust \\
\hline \multirow{2}{*}{ (i)nai } & (si) & len & charícha \\
\hline
\end{tabular}




$\begin{array}{lllll}\text { - (i)nai len so } & \text { te jan } & \\ \text { neg. Obj. dir. 3pl. qué } & \text { sub. comer } \\ \text { no tienen qué } & \text { comer } & & \end{array}$

\begin{tabular}{|c|c|c|c|c|c|}
\hline \multicolumn{6}{|c|}{$\mathrm{O}$} \\
\hline Negación & \multicolumn{2}{|c|}{$+\mathrm{P}$} & \multicolumn{3}{|c|}{ Obj dir } \\
\hline \multirow[t]{4}{*}{ Adv neg } & \multirow{2}{*}{\multicolumn{2}{|c|}{ FMV }} & \multicolumn{3}{|c|}{ ODep } \\
\hline & & & Obj dir & Subordinación & $+\mathrm{P}$ \\
\hline & Centro & Mod & \multirow{2}{*}{ Pron interrogat } & \multirow[t]{2}{*}{ Part sub } & \multirow[t]{2}{*}{$\mathrm{V}$} \\
\hline & $(\mathrm{V})$ & Pron acus & & & \\
\hline (i)nai & (si) & len & so & te & jan \\
\hline
\end{tabular}

\section{Consideración final}

Como hemos señalado, este trabajo consta de dos partes. Las conclusiones finales y sus proyecciones se entregarán en la segunda parte. Con todo, nos parece pertinente señalar que hemos cumplido con nuestro objetivo de presentar los aspectos más prominentes de la morfología nominal y verbal del rromané, los aspectos focales del marco de referencia tagmémico, la metodología que seguimos paso a paso para el trabajo de campo y los análisis, y, como foco, la explicitación de los tagmemas instanciadores y modificadores presentes en la Frase Modificada Sustantiva y Frase Modificada Verbal.

En nuestro segundo artículo y final, explicitaremos los tipos de Frases restantes, los tagmemas y construcciones presentes en el nivel de la Oración, así como la evaluación del marco teórico empleado, y las conclusiones finales que se extraen de esta investigación.

\section{Bibliografía}

Elson, B. y V. Pickett. (1964). An Introduction to Morphology and Sintax. Ciudad de México: Summer Institute Linguistics.

González A. y Salamanca, G. (2001). «Descripción fonológica del romané de Chile», en Revista de Filología y Lingüística de la Universidad de Costa Rica Vol. XXVIII(1), pp. 177-197.

Grant, A. (1994). Romani: A Conspectus. Notes o Romani Lenguaje and Linguistics. Bradford: University of Bradfor.

Gutiérrez, S. (1997). La Oración y Sus Funciones. Madrid: Arco Libros. 
Hancock, I. (1993). Grammar of Vlax Romani. Texas: Romanestan Publications.

Lizarralde, D. \& Salamanca G. (2010). "Morfología de los Adjetivos Demostrativos en el Rromané Jorajané de Chile", en Literatura y Lingüística 21, pp. 109-126.

Pike, K. (1958). “On tagmemes, née gramemes”, en International Journal of American Linguistics 24, pp. 273-278.

Salamanca, G. (2003). Morfología nominal y verbal del romané, lengua de los gitanos de Chile, Tesis para optar al grado de Doctor en Lingüística, Universidad de Concepción.

(2004). «Notas sobre la morfología verbal del rromané, lengua de los gitanos de Chile (1)», en Logos $\mathrm{N}^{\circ}$ 14, pp. 73-96.

(2005). «Vitalidad lingüística y cultural en un mundo globalizado: El caso del rromané, frente a las lenguas vernáculas chilenas», en Sociedad Hoy No 8-9.

(2006). «Notas sobre la morfología nominal del rromané, lengua de los gitanos de Chile (II)», en Alpha $\mathrm{N}^{\circ}$ 22, pp. 209-222.

(2008). «Notas sobre la morfología nominal del rromané, lengua de los gitanos de Chile (I)», en Literatura y Lingüística No 19, pp. 211-233.

y González, A. (1999). "Gitanos de Chile: Un acercamiento etnolingüística”, en Atenea No 480, pp. 141-172.

y Lizarralde, D. (2008). «Propuesta de un grafemario para el rromané jorajané, lengua hablada por los gitanos de Chile (1)», en Universum $\mathrm{N}^{\circ}$ 23(1), pp. 226-247.

Salas, A. (1978). Semantic Ramifications of the Category of Person in the Mapuche Verb. Dissertation submitted to the Faculty of the Graduate School of State University of New York at Buffalo. Ms. Publicado después en Ann Arbor: University Microfilms International.

Samarin, W. (1967). Field Linguistics: A Guide to Linguistic Field Work. New York: Holt, Rinehart and Wiston.

Soravia, G. (1984). «La lengua, rastro de una larga peregrinación», en El Correo de la UNESCO XXXVII, pp. 21-23.

Sotomayor, C. (1993). «Gitanos: 1000 años de viajes en el cuerpo», en El Canelo 41, pp. 14-21. 